\title{
On-Line Signature Matching Based on Hilbert Scanning Patterns
}

\author{
Alireza Ahrary ${ }^{1,2}$, Hui-ju Chiang ${ }^{3}$, and Sei-ichiro Kamata ${ }^{4}$ \\ 1 Fukuoka Industry, Science and Technology Foundation, Japan \\ ${ }^{2}$ Information, Production and Systems Research Center, Waseda University, Japan \\ 3 Department of Computer Science, National Tsing Hua University, Taiwan \\ ${ }^{4}$ Graduate School of Information, Production and Systems, \\ Waseda University, Japan
}

\begin{abstract}
Signature verification is a challenging task, because only a small set of genuine samples can be acquired and usually no forgeries are available in real application. In this paper, we propose a novel approach based on Hilbert scanning patterns and Gaussian mixture models for automatic on-line signature verification. Our system is composed of a similarity measure based on Hilbert scanning patterns and a simplified Gaussian mixture model for decision-level evaluation. To be practical, we introduce specific simplification strategies for model building and training. The system is compared to other state-of-the-art systems based on the results of the First International Signature Verification Competition (SVC 2004). Experiments are conducted to verify the effectiveness of our system.
\end{abstract}

Keywords: Hilbert scanning patterns, Gaussian mixture model, Hilbert scanning distance.

\section{Introduction}

Biometric user recognition techniques are currently undergoing large-scale deployment. Among biometrics, they can be categorized into two classes of methods: physiological and behavioral biometrics. Physiological traits including hand and face geometry, are based on the measurement of biological properties of users by devices like digital cameras and scanners. In contrast to physiological systems, behavioral biometrics, such as recognition of subjects by voice or signatures, require an explicit action to be performed by individuals and thus imply awareness [1. Signature verification is one of behavioral biometrics which has been an intense research area because of the social and legal acceptance and widespread use of the written signature as a personal authentication method.

Depending on the acquisition process, automatic signature verification systems are divided into two categories: on-line and off-line. In early off-line cases, signatures are captured until the writing process is finished, thus only static images are available. Recently, more researches focus on the online signature verification, where signatures are acquired during the writing process with a special instrument, such as digital tablet. In fact, dynamic information is available 
in the case of online signature verification, such as velocity, acceleration and pressure which is more difficult to imitate than the static shape of signature. Therefore, online signature verification can usually achieve better performance than the offline instance. Many different approaches have been considered in the literature in order to extract discriminative information from on-line signature data. The existing methods can broadly be divided into two classes; i) Featurebased approaches, in which a holistic vector representation consisting of a set of global features is derived from the signature trajectories [2]. ii) Function-based approaches, in which time sequences describing local properties of the signature are used for recognition $[3$.

Function-based approaches can be classified into local and regional methods. In local approaches, the time functions of different signatures are directly matched by using elastic distance measures such as Dynamic Time Warping (DTW) 44. In regional methods, the time functions are converted to a sequence of vectors describing regional properties. One of the most popular regional approaches is the method based on Hidden Markov Models (HMM) [5]. Generally, the DTW is regarded as a popular method, but it usually suffers from the following two drawbacks; Heavy computational load and Warping forgeries [6]. The first one makes the DTW time-consuming while the second makes the verification more difficult. On the other hand, the HMM has the capability to perform stochastic matching for a model of signature using a sequence of probability distributions of the features along the signature. Practically, the HMM has been employed in the filed of online signature verification for two decades and has achieved some success. However, the HMM also has its limitations. Its poor discriminative power is fatal which limits its application on the signature verification [7]. These models focus on local properties of signatures such as local moving direction and shape curvature. Global features like writing time or signature length may be incorporated to speed up verification procedure and improve accuracy.

In the practical applications, users may feel uncomfortable if required to provide lots of samples. Only small set of signature samples can be acquired [8]. This demands the ideal signature verification system to be simple because complex system usually needs large training sample set. On the other hand, many existing systems require false samples, i.e., forgery signatures, for system training. But it is actually hard to collect forgery signatures for every user in practice. So the practical system would be more applicable if it uses forgery samples as few as possible.

Due to the above-mentioned two considerations, we proposed a novel method for automatic signature verification system based on Hilbert Scanning (HS) patterns and GMM. The paper is organized as follows. Section 2 and Section 3 detail the theoretical background of the Hilbert Scanning patterns and the statistical modeling based on GMM. In Section 4 gives an overview of the proposed system and its main components. The evaluation of decision-level combination strategies are presented in Section 5. Experimental procedure and results are given in Section 6. Finally in Section 7 we present our conclusions and suggestions for future work. 


\section{Hilbert Scanning Patterns}

Space-filling curve is one-to-one mapping between $\mathrm{N}$-dimensional (N-D) space and one-dimensional (1-D) space. By mapping each point in a N-D space into a 1-D space for a single point, the complex multidimensional access methods can be transformed into simple 1-D processing methods [9]. For this advantage, a lot of research works have been done and there have been many space-filling curves proposed. In 1980, the mathematician G. Peano presented a family of curves [10], such as raster curve, $\mathrm{Z}$ curve and Hilbert curve. The Hilbert curve is a locus of points in N-D space. For its special attributes, it becomes an analytical solution of a space-filling curve. In 1891 Hilbert made a curve having the space-filling property in 2-D space (Hilbert curve).

The N-D Hilbert scanning is regarded as a mapping from a point in N-D space to a point in 1-D space using the Hilbert curve. Quinqueton et al. 11] proposed an algorithm for computing the scan by a recursive procedure. However, in general the recursive procedure presents a drawback for high speed computation and hardware implementation. On the other hand, a continuous one-to-one mapping algorithm is considered by Butz [12] but this algorithm is complex. Kamata first discussed a fast sequential algorithm with look-up tables requiring little memory 13 . He also proposed an effective method for constructing look-up tables of N-dimensional Hilbert scanning derived from reflected binary gray codes in order to improve the performance, and also a simple non-recursive algorithm using the look-up tables which expands the algorithm for 2-D space to one for N-dimensional space. Fig. 1 shows how the Hilbert scan transforms the two-dimensional space to one-dimensional sequence.

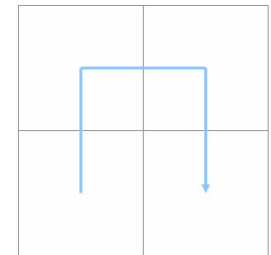

Hilbert Scan in $2 \times 2$

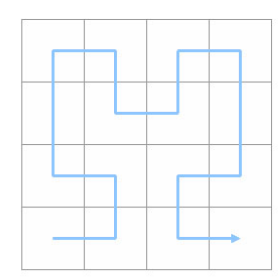

Hilbert Scan in $4 \times 4$

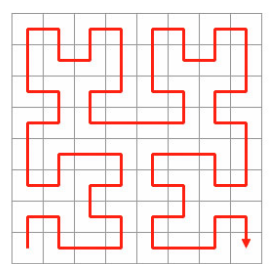

Hilbert Scan in $8 \times 8$

Fig. 1. The Hilbert scanning sequence

The Hilbert scan has two important features; first, extracting a group of neighboring pixels having similar intensities is easier by using Hilbert scan than other scan techniques such as raster scan, row-prime scan, Morton scan, and second is Hilbert scan preserves the coherence in a 2-D space. That is, if the Euclidean distance between two points in the 2-D space is small, then the scanning length between the same pair of points in the 1-D sequence is also small.

The HS patterns using in this paper is the 1-D sequence transformed from the 2-D space, and we use the patterns to compute the similarity measure. 


\section{Gaussian Mixture Models}

GMM is a well known and so much referenced technique for pattern recognition. GMM theory has been known for ages, but it was not till Expectation Maximization algorithm was developed [16] that it become a useful technique for pattern recognition.

GMM can be thought of as a single state HMM, which means HMM training methods can be applied to GMM with very little or no modification. GMM are widely-used statistical models in many pattern recognition applications. They are a versatile modeling tool as they can be used to approximate any probability density function given a sufficient number of components, and impose only minimal assumptions about the modeled random variables. A GMM is a weighted combination of multiple Gaussians. A distinct property of GMM is its power to approximate any probability density. Let us denote a component GMM by $\Theta=\left\{\left(\alpha_{l}, \mu_{l}, \Sigma_{l}\right): l=1, \ldots, M\right\}$. It has the general form as follows;

$$
p(x \mid \Theta)=\sum_{l=1}^{M} \alpha_{l} \cdot p\left(x \mid \mu_{l}, \Sigma_{l}\right)
$$

where $\mathrm{x}$ is an n-dimensional feature vector, $\alpha_{l}$ is the weighted coefficient with $\alpha_{l}=p(l \mid \Theta), \Sigma \alpha_{l}=1, \mu_{l}$ and $\Sigma_{l}$ are the mean vector and the covariance matrix of the $l$-th Gaussian component, respectively. Each component's distribution is an n-variable Gaussian function. To avoid GMM learning from being stuck into local extreme, K-means clustering is usually applied for "good" initialization. We may choose the best combination with small intra-class distance and large inter-class distance by exhaustive search. Note that the feature vector does not have the same unit in each dimension. We should normalize each dimension before computing the distance matrix between two feature vectors.

For getting this model for each user, Expectation - Maximization (EM) 16] algorithm has been used. EM provides an easy way to estimate the three elements for GMM in an iterative mode. The elements of the models have to be initialized. Random selection from the training data have been used to set up the mean vectors, covariance matrix has been initialized as the unit matrix, and each weighted coefficient is defined as 1/M. After initialization, EM iterative algorithm is run until getting the threshold.

\section{System Overview}

\subsection{Preprocessing}

On-line signature always contains associated dynamic information [5], such as: (i) Position in x-axis. (ii) Position in y-axis. (iii) Pressure applied by the pen. (iv) Azimuth angle of the pen with respect to the tablet. (v) Altitude angle of the pen with respect to the tablet.

A signature acquisition process on a restricted size frame is assumed [15]. As a result, users are supposed to be consistent in size and writing dynamics. Moreover, a geometric normalization consisting of position normalization followed by 
rotation alignment is applied. In this paper, each signature is normalized on the position and scale firstly.

\subsection{Feature Points Extraction}

Only coordinate trajectories are considered in the feature extraction process. The feature points are defined as the peaks and valleys of the signal 6 . We first define a rise-distance, denoted by " $\mathrm{r}$ ", as the amplitude from a valley to the following peak. Similarly we define a drop-distance, denoted by "d", as the amplitude from a peak to the following valley. For any peak (or valley), a rise distance can be computed at one side of the curve, while a drop-distance can be computed at the other side of the curve. The peak or valley is marked as a function point only if $r \geq h_{o}, d \geq h_{0}$ condition is satisfied; where $h_{0}$ is defined as a threshold. Small ripples are not considered as feature points. This is because small ripples are unreliable most of the time. In the project, the threshold $h_{0}$ is chosen as one pixel. Hence any ripples with rise or drop distance less than $h_{0}$ will not the counted as feature points. Our simple feature points extracting function can identify the important peaks and valleys along the signal, while excluding the small ripples, as shown in Fig. 2.

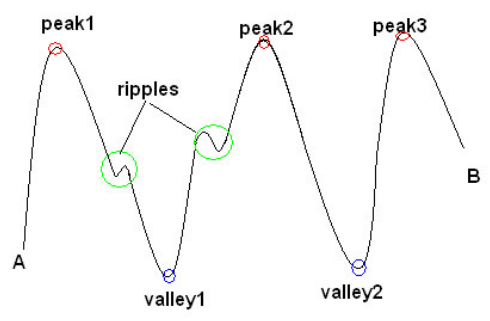

Fig. 2. Examples of peaks, valleys and ripples

\subsection{Data Training Procedure}

We have two training procedure for Hilbert scanning patterns and Gaussian mixture models respectively. First, we use the Hilbert scanning algorithm to convert the feature points in 2-D space into 1-D sequence. Then, the training procedure of Hilbert scanning patterns consist first 10 genuine signatures for each user and compute the Hilbert scanning distance between each two signatures to represent the Hilbert threshold of each user. In the GMM training, we also apply first 10 signatures of each user for modeling. Every single user will have his/her own model, we will use $\Theta$ as the notation to reference each user GMM as defined in eq.1.

\section{Evaluation}

\subsection{Hilbert Scanning Distance}

In this paper, we use the Hilbert Scanning Distance which is a distance measure using the Hilbert curve to measure the similarity. Hilbert Scanning Distance 
can be viewed as a special Hausdorff distance extended to the 1-D space. We use the Hilbert curve to convert the two-dimensional (2-D) searching space into a one-dimensional (1-D) sequence. An important feature of Hilbert scanning is that it can preserve the coherence in the 2-D image. Thus, the distance between two points in the $2-\mathrm{D}$ space can be migrated into a $1-\mathrm{D}$ sequence. In the 1$\mathrm{D}$ sequence, we only need to consider front and back neighborhood instead of multi-neighborhood in the 2-D space, which means searching many fewer points when computing the distance.

Assume that we are given two finite point sets: $A=\left\{a_{1}, \ldots, a_{I}\right\}$ and $B=$ $\left\{b_{1}, \ldots, b_{J}\right\}$ such that each point $a \in A$ and $b \in B$ has integer coordinates in the 2-D space. We firstly use Hilbert scanning to convert them to new sets $S=\left\{s_{1}, \ldots, s_{I}\right\}$ and $T=\left\{t_{1}, \ldots, t_{J}\right\}$ in the 1 -D sequence, respectively. Then, the directed Hilbert scanning distance from $A$ to $B, h_{h s d}(A, B)$ is computed by;

$$
h_{h s d}(A, B)=\frac{1}{I} \sum_{i=1}^{I} \rho\left(\min _{j}\left\|s_{i}-t_{j}\right\|\right)
$$

where $\|\cdot\|$ is the Euclidean norm distance in the 1-D space and function $\rho$ is defined as;

$$
\rho(x)=\left\{\begin{array}{l}
x(x \leq \tau) \\
\tau(x>\tau)
\end{array}\right.
$$

where $\rho$ is called threshold elimination function and $\tau$ is a threshold predefined. We also can obtain the directed Hilbert scanning distance from $B$ to $A$, $h_{h s d}(B, A)$ similarly and Hilbert scanning distance is defined by;

$$
H_{h s d}(A, B)=\max \left(h_{h s d}(A, B), h_{h s d}(B, A)\right)
$$

\subsection{GMM Similarity}

GMM is a weighted combination of multiple Gaussians. A distinct property of GMM is its power to approximate any probability density. Denote a component GMM by the notation. $\Theta=\left\{\left(\alpha_{l}, \mu_{l}, \Sigma_{l}\right): l=1, \ldots, M\right\}$. It has the general form as follows;

$$
p(x \mid \Theta)=\sum_{l=1}^{M} \alpha_{l} \cdot p\left(x \mid \mu_{l}, \Sigma_{l}\right)
$$

Every single user will have his/her own model, and we will compute the similarity between the model and training data to get a GMM threshold of each user for the testing procedure.

\section{Experiments}

\subsection{Database Description}

There are not many signature databases publicly available at the moment for research purposes. As a result, the common practice in on-line signature recognition research is to evaluate the proposed recognition strategies on small data sets 
acquired at the different research laboratories. In this environment, the First International Signature Verification Competition (SVC 2004) has been organized 14 providing a common reference for system comparison on the same signature data and evaluation protocol.

Development corpus of the extended task (including coordinate and timing information, pen orientation and pressure) is used in the experiments that follow. The database 1 used in this experiment consists 5 sets of signatures, the database 2 consists of 40 sets of signatures (Task1 in SVC2004), and the database 3 includes 40 sets of signatures (Task2 in SVC2004). Each set contains 20 genuine signatures from one contributor (acquired in two separate sessions) and 20 skilled forgeries from five other contributors.

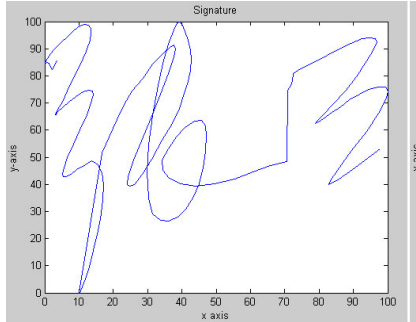

(a)

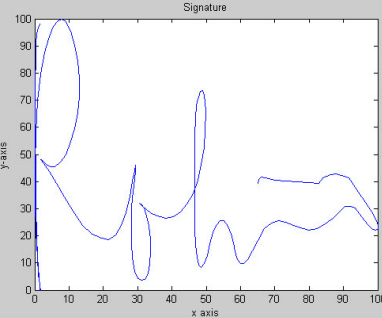

(b)

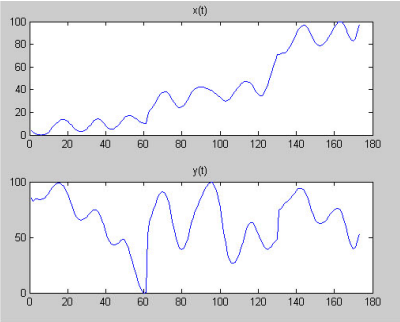

(c)

Fig. 3. (a) shows the Chinese sample of the dataset, (b) shows the English sample of the dataset and $(\mathrm{c})$ shows the trajectories of $\mathrm{x}(\mathrm{t})$ and $\mathrm{y}(\mathrm{t})$ of the dataset

The signatures are mostly in either English or Chinese. Some examples are shown in Fig. 3 for two different targets of the data set. Plots of the coordinate trajectories are also given.

\subsection{Experimental Results}

The following tables show the experimental results of the system. The results in terms of the equal error rate (EER) obtained by GMM and proposed method are presented in Table 1. This experiment uses 10 genuine signatures for training, the last 10 genuine signatures and 20 skilled forgery signatures for testing. For skilled signatures, the proposed method acquires the better equal error rate than GMM method.

Random forgeries are also taken into consideration here. We randomly select 20 signatures from other subjects in database for each signer as imposter data. Table 2 shows another experimental result which uses 10 genuine signatures for training, the last 10 genuine signatures and 20 random forgery signatures for testing.

Comparison between our system and the best 5 system competing in SVC2004 task1 and task2, which are following the same experimental protocol, is also given in Table 3 and Table 4. From Table 3 and Table 4, we can see that proposed 
Table 1. Error rate of methods for skilled forgeries (Training data=10 samples, testing data $=10$ genuine +20 skilled forgeries)

\begin{tabular}{|c|c|c|c|}
\hline Method $\backslash$ EER & Database1 & Database2 & Database3 \\
\hline GMM method & $16 \%$ & $15.58 \%$ & $14.41 \%$ \\
\hline Proposed method & $4.6 \%$ & $6.08 \%$ & $5.83 \%$ \\
\hline
\end{tabular}

Table 2. Error rate of methods for random forgeries (Training data=10 samples, testing data $=10$ genuine +20 random forgeries)

\begin{tabular}{|c|c|c|c|}
\hline Method $\backslash$ EER & Database1 & Database2 & Database3 \\
\hline GMM method & $12.6 \%$ & $9.0 \%$ & $11.1 \%$ \\
\hline Proposed method & $4.6 \%$ & $3.98 \%$ & $3.25 \%$ \\
\hline
\end{tabular}

Table 3. Error rate of methods using database2 (Training data=10 samples, testing data $=10$ genuine +20 forgeries)

\begin{tabular}{ccc}
\hline Team id & $\begin{array}{c}10 \text { genuine signatures } \\
+20 \text { skilled forgeries }\end{array}$ & $\begin{array}{c}10 \text { genuine signatures } \\
\text { 20 random forgeries }\end{array}$ \\
\hline 106 & $5.50 \%$ & $3.65 \%$ \\
Proposed method & $6.08 \%$ & $3.98 \%$ \\
126 & $6.45 \%$ & $3.49 \%$ \\
124 & $7.33 \%$ & $2.93 \%$ \\
115 & $9.80 \%$ & $2.90 \%$ \\
\hline
\end{tabular}

Table 4. Error rate of methods using database 3 (Training data=10 samples, testing data $=10$ genuine +20 forgeries)

\begin{tabular}{ccc}
\hline Team id & $\begin{array}{c}10 \text { genuine signatures } \\
+20 \text { skilled forgeries }\end{array}$ & $\begin{array}{c}10 \text { genuine signatures } \\
\text { 20 random forgeries }\end{array}$ \\
\hline Proposed method & $5.83 \%$ & $3.25 \%$ \\
$219 \mathrm{~b}$ & $6.90 \%$ & $3.02 \%$ \\
$219 \mathrm{c}$ & $6.91 \%$ & $3.47 \%$ \\
206 & $6.96 \%$ & $2.93 \%$ \\
229 & $7.64 \%$ & $4.45 \%$ \\
\hline
\end{tabular}

method gives a better performance in database 2 (Task 1 in SVC 2004) and also performs well both in skilled forgeries and random forgeries in database 3 (Task 2 in SVC 2004).

\section{Conclusion}

In this paper, we proposed a novel algorithm based on Hilbert scanning patterns and Gaussian mixture models for signature verification system. Proposed method 
has two main procedures including training procedure and testing procedure. For training procedure, we compute the Hilbert threshold and GMM threshold representing the similarity. In testing procedure, we proposed a two-stage model to verify the signatures by Hilbert threshold and GMM threshold. The system shows that we can use the fewer features to get the better performance, yielding not only high recognition rate but also less processing time on public database SVC2004.

The possible improvement on this work mainly lies in the combination of the decision. The decision making based on the combined verification is straightforward which need more investigation for improvement. We consider that the threshold variation of every parameter is related to this problem. Examination of a policy to equalize a threshold variation every such parameter is a problem.

\section{Acknowledgment}

This work was supported by a grant of Knowledge Cluster Initiative 2nd stage implemented by Ministry of Education, Culture, Sports, Science and Technology (MEXT).

\section{References}

1. Vielhauer, C.: A behavioural biometric. Public Service Review: EUROPEAN UNION, 113-115 (2005)

2. Ketabdar, H., Richiardi, J., Drygajlo, A.: Global feature selection for on-line signature verification. In: Proc. of 12th Int'l Graphonomics Society Conf. (2005)

3. Fairhurst, M.C.: Signature verification revisited: Promoting practical exploitation of biometric technology. IEE Electronics and Communication Engineering Journal 9(6), 273-280 (1997)

4. Faundez-Zanuy, M.: On-line signature recognition based on VQ-DTW. Pattern Recognition 40, 981-992 (2007)

5. Fierrez, J., Ortega-Garcia, J., Ramos, D., Gonzalez-Rodriguez, J.: HMM-based on-line signature verification: feature extraction and signature modeling. Pattern Recognition Letters 28(16), 2325-2334 (2007)

6. Feng, H., Wah, C.C.: Online Signature Verification Using a New Extreme Points Warping Technique. Pattern Recognition Letters 24(16), 2943-2951 (2003)

7. Trentin, E., Gori, M.: A Survey of Hybrid HMM/ANN Models for Automatic Speech Recognition. Neurocomputing 37, 91-126 (2001)

8. Zou, M., Tong, J., Liu, C., Lou, Z.: On-line signature verification using local shape analysis. In: Proc. of the 7th Int'l Conf. Document Analysis and Recognition, pp. 314-318 (2003)

9. Kamata, S., Kawaguchi, E.: A method of computing Hilbert curves in two and three dimensional spaces. Int'l Conf. Pattern Recognition, 93-97 (1994)

10. Peano, G.: Sur une courbe qui remplit touteune aire plane. Math. Ann. 36, 157-160 (1890)

11. Quinqueton, J., Berthod, M.: A locally adaptive peano scanning algorithm. IEEE Trans. Pattern Anal. Mach. Intell., PAMI 3(4), 409-412 (1981) 
12. Butz, A.: Convergence with Hilbert's space filling curve. Computer and System Science 3(2), 128-146 (1969)

13. Kamata, S., Kawaguchi, E.: A method of computing Hilbert curves in two and three dimensional space. Trans. IEICE J74-D-II, 1217-1226 (1991)

14. Yeung, D.Y., Chang, H., Xiong, Y., George, S., Kashi, R., Matsumoto, T., Rigoll, G.: SVC2004: First international signature verification competition. In: Proc. Intl. Conf. Biometric Authentication, pp. 16-22 (2004)

15. Fierrez-Aguilar, J., Alonso-Hermira, N., Moreno-Marquez, G., Ortega-Garcia, J.: An off-line signature verification system based on fusion of local and global information. In: Maltoni, D., Jain, A.K. (eds.) BioAW 2004. LNCS, vol. 3087, pp. 295-306. Springer, Heidelberg (2004)

16. Bilmes, J.: A Gentle Tutorial of the EM Algorithm and its Application to Parameter Estimation for Gaussian Mixture and Hidden Markov Models. Technical Report ICSI-TR-97-021 (1998) 